University of Nebraska - Lincoln

DigitalCommons@University of Nebraska - Lincoln

June 2006

\title{
Perturbation of magnetostatic modes observed by ferromagnetic resonance force microscopy
}

\author{
R. Urban \\ California Institute of Technology, MC 114-36, Pasadena, California
}

A. Putilin

California Institute of Technology, MC 114-36, Pasadena, California

P.E. Wigen

California Institute of Technology, MC 114-36, Pasadena, California

Sy_Hwang Liou

University of Nebraska-Lincoln, sliou@unl.edu

M.C. Cross

California Institute of Technology, MC 114-36, Pasadena, California

See next page for additional authors

Follow this and additional works at: https://digitalcommons.unl.edu/physicsliou

Part of the Physics Commons

Urban, R.; Putilin, A.; Wigen, P.E.; Liou, Sy_Hwang; Cross, M.C.; Hammel, P.C.; and Roukes, M.L.,

"Perturbation of magnetostatic modes observed by ferromagnetic resonance force microscopy" (2006).

Si-Hwang Liou Publications. 90.

https://digitalcommons.unl.edu/physicsliou/90

This Article is brought to you for free and open access by the Research Papers in Physics and Astronomy at DigitalCommons@University of Nebraska - Lincoln. It has been accepted for inclusion in Si-Hwang Liou Publications by an authorized administrator of DigitalCommons@University of Nebraska - Lincoln. 


\section{Authors}

R. Urban, A. Putilin, P.E. Wigen, Sy_Hwang Liou, M.C. Cross, P.C. Hammel, and M.L. Roukes 


\title{
Perturbation of magnetostatic modes observed by ferromagnetic resonance force microscopy
}

\author{
R. Urban, ${ }^{1}$ A. Putilin, ${ }^{1}$ P. E. Wigen,,${ }^{1,2}$ S.-H. Liou, ${ }^{3}$ M. C. Cross, ${ }^{1}$ P. C. Hammel, ${ }^{2}$ and M. L. Roukes ${ }^{1}$ \\ ${ }^{1}$ Condensed Matter Physics, California Institute of Technology, MC 114-36, Pasadena, California 91125, USA \\ ${ }^{2}$ Department of Physics, Ohio State University, Columbus, Ohio 43210, USA \\ ${ }^{3}$ Department of Physics and Astronomy, University of Nebraska, Lincoln, Nebraska 68588, USA \\ (Received 8 February 2006; revised manuscript received 9 May 2006; published 14 June 2006)
}

\begin{abstract}
Magnetostatic modes of yttrium iron garnet (YIG) films are investigated by ferromagnetic resonance force microscopy. A thin-film "probe" magnet at the tip of a compliant cantilever introduces a local inhomogeneity in the internal field of the YIG sample. This influences the shape of the sample's magnetostatic modes, thereby measurably perturbing the strength of the force coupled to the cantilever. We present a theoretical model that explains these observations; it shows that the tip-induced variation of the internal field creates either a local "potential barrier" or "potential well" for the magnetostatic waves. The data and model together indicate that local magnetic imaging of ferromagnets is possible, even in the presence of long-range spin coupling, through the introduction of localized magnetostatic modes predicted to arise from sufficiently strong tip fields.
\end{abstract}

DOI: $10.1103 /$ PhysRevB.73.212410

PACS number(s): 76.50.+g, 07.55.-w, 07.79.Pk, 41.20.Gz

In the past decade, the possibility of using magnetic degrees of freedom in electronic devices has attracted the attention of the semiconductor industry. There are numerous proposals to incorporate spin degrees of freedom into electronics creating spin-electronic or spintronic devices. ${ }^{1}$ These devices require a thorough understanding of the magnetization dynamics on the submicrometer scale. Magnetic resonance force microscopy (MRFM) has proven itself to be a three-dimensional nondestructive imaging technique that can be applied to electron ${ }^{2-4}$ and nuclear ${ }^{5}$ resonance experiments. Ferromagnetic resonance force microscopy (FMRFM $)^{6-9}$ is a variation of MRFM that enables the three-dimensional, highsensitivity and high-resolution qualities of MRFM to be applied in the characterization of the dynamic magnetic properties of ferromagnetic thin films and multilayers. Recent publications have shown the ability of FMRFM to determine the nature and magnitude of terms contributing to the internal field, ${ }^{7}$ dispersion relations of magnetostatic modes, ${ }^{6,8,10,11}$ and relaxation processes in micrometer-sized samples. ${ }^{12}$ Ferromagnetically coupled systems pose unique challenges for magnetic resonance imaging due to the strong coupling between the spins. The resulting magnetostatic/exchange resonance modes involve spins occupying the entire sample, not just those within the resonance slice. ${ }^{10}$ Therefore, local imaging capability of MRFM is lost in the case of ferromagnetic samples.

In this paper, we report a direct observation of the modification of the magnetostatic mode amplitudes due to the local perturbation of the internal field. The localized field is produced by the sharp magnetic tip when it is scanned across the surface of yttrium iron garnet (YIG) samples. The experimental results are in excellent agreement with theoretical predictions. In addition, highly localized modes can be excited to recover, in part, local imaging capabilities. The localized magnetic modes will provide an exciting opportunity to investigate static and dynamic magnetic properties of micrometer-sized defects (imperfections) embedded in ferromagnetic samples without the interference of edge defects.

The experiments were performed using an ambient FMRFM system in the parallel force geometry ${ }^{10}$ (external dc field is applied parallel to the sample normal). The sample is attached to a microstrip resonator having a resonance frequency of $7.4 \mathrm{GHz}$. The silicon cantilever's resonance frequency, the spring constants, and the ambient $Q$ factor are $17.8 \mathrm{kHz}, 0.4 \mathrm{~N} / \mathrm{m}$, and 90, respectively, yielding a force sensitivity of $6 \times 10^{-14} \mathrm{~N}$ for $1 \mathrm{~Hz}$ bandwidth. The cantilever displacement is monitored at $850 \mathrm{~nm}$ by a fiber optic interferometer. The cantilever tip was coated with a $250 \mathrm{~nm} \mathrm{CoPt}$ film $\left(M_{s}=800 \mathrm{emu} / \mathrm{cm}^{3}\right)$ and annealed in an external magnetic field of $80 \mathrm{kOe}$ oriented along the tip axis of the cantilever. This resulted in a coercive field of $10 \mathrm{kOe} .{ }^{13}$ The magnetic tip generates a localized tip field, $h_{\text {tip }}$, which can be aligned either parallel $\left(h_{\text {tip }}>0\right)$ or antiparallel $\left(h_{\text {tip }}<0\right)$ to the external dc field, $H_{\text {ext }}$. Based on a model of the magnetic tip, the strength of the tip field and the tip field gradient at the tip-sample separation $z=1 \mu \mathrm{m}$ were estimated to be $80 \mathrm{G}$ and $50 \mathrm{G} / \mu \mathrm{m}$ for $h_{\text {tip }}>0$. These parameters translate into a magnetic sensitivity of $10^{6}$ Bohr magnetons.

Typical measured spectra obtained on the $20 \times 80 \mu \mathrm{m}^{2}$ YIG sample are shown in Fig. 1. The magnetostatic modes are labeled by $\left(n_{x}, n_{y}\right)$, where $n_{x}$ and $n_{y}$ represent the number of antinodes along the sample width and length, respectively. In the presence of a uniform bias field and a homogeneous driving rf field, only modes with odd values of $n_{x}$ and $n_{y}$ have a net dipole moment that will couple to the rf field. ${ }^{14}$

$h_{\text {tip }}>0$. To investigate the effects of this perturbation quantitatively, we first discuss the case in which the magnetic field of the tip magnet adds to the bias magnetic field. For a weak tip field $\left(h_{\text {tip }} \approx 0\right)$ applied at the center of the sample, the mode amplitudes are observed to decrease monotonically with increasing $n_{x}$ and $n_{y}$. Such a spectrum is shown by the dashed line in Fig. 1(a) for $z=10 \mu \mathrm{m}$. At that distance, the magnetic field generated by the tip magnet is on the order of $2 \mathrm{G}$. As the tip approaches the sample surface, the magnetic tip field increases. This results in a dramatic change of the measured spectra as illustrated by the solid line in Fig. 1(a). There are two main features to be noted: (i) the spectrum is shifted to a lower resonance field by $(8 \pm 1) \mathrm{G}$ and (ii) the amplitude of modes $(1,5)$ and $(1,9)$ is enhanced relative to modes $(1,3),(1,7)$, and $(1,11)$. The resonance field shift is due to the spatially averaged tip field, which adds to the external field. 


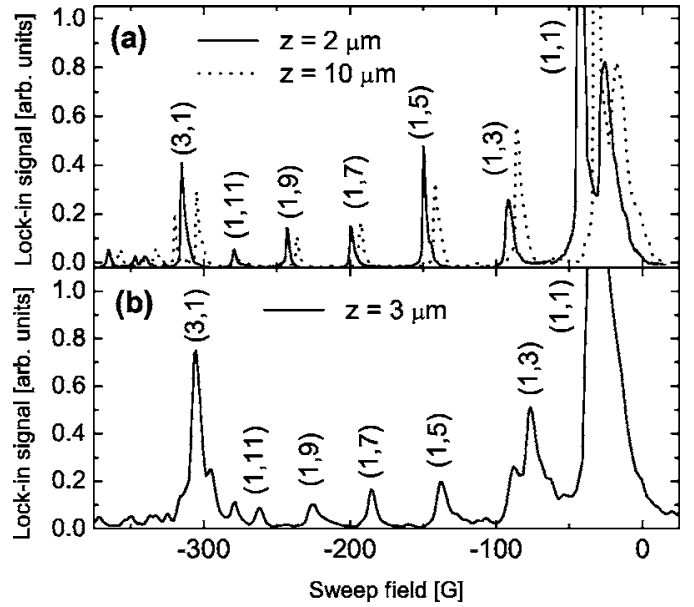

FIG. 1. Typical FMRFM spectra measured in the center $(y$ $=0 \mu \mathrm{m}$ ) of the $20 \times 80 \mu \mathrm{m}^{2}$ sample for (a) $h_{\text {tip }}>0$ and (b) $h_{\text {tip }}<0$, respectively. The dashed and the solid lines in (a) correspond to the tip-sample separation of 10 and $2 \mu \mathrm{m}$, respectively. Zero sweep field corresponds to the resonance field of the homogeneous mode $\omega / \gamma+4 \pi M_{s}=4.4 \mathrm{kG}$. The modes are labeled by $\left(n_{x}, n_{y}\right)$, where $n_{x}$ and $n_{y}$ represent the number of half-wavelengths along the sample width and length, respectively.

To demonstrate the subtle details of the effect of the increasing tip field, the force intensity for modes $(1,3)$ and $(1,5)$ is plotted in Fig. 2 as a function of the tip-sample separation. As the tip field gradient increases with decreasing distance, the FMRFM signal increases. However, the intensity of the $(1,3)$ mode decreases dramatically for $z<3 \mu \mathrm{m}$ and at $z=2.5 \mu \mathrm{m}$ its intensity becomes even lower than the intensity of the $(1,5)$ mode. For $z<2 \mu \mathrm{m}$, the intensity of the $(1,5)$ mode decreases as well. These effects can be explained by the model proposed below.

$h_{\text {tip }}<0$. By reversing the orientation of the applied magnetic field without reversing $h_{\text {tip }}$, the magnetic field of the tip subtracts from the homogeneous bias field. For a tip-sample separation below $2 \mu \mathrm{m}$, the modes $(1,3),(1,7)$, and $(1,11)$ are now stronger compared to modes $(1,5)$ and $(1,9)$, see Fig. 1(b). This is exactly opposite to what is observed for $h_{\text {tip }}$ $>0$. Table I summarizes the intensity ratios of the subsequent resonant modes when the tip field is weak and the magnetostatic modes are practically unperturbed, $h_{\text {tip }} \approx 0$

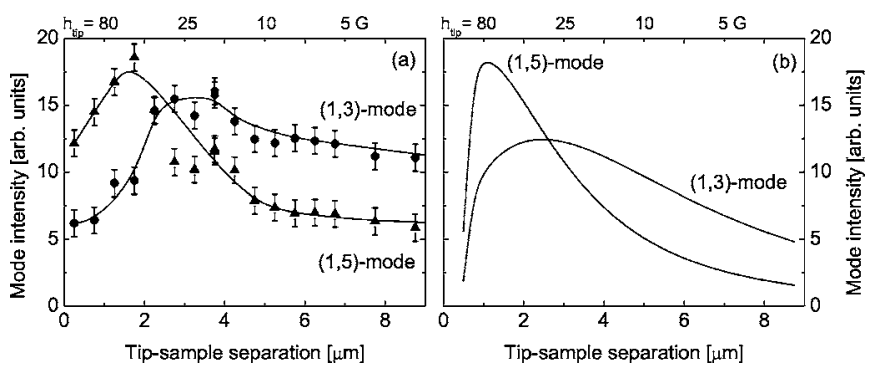

FIG. 2. (a) Experimental and (b) theoretical mode intensity of the $(1,3)$ mode (circles) and $(1,5)$ mode (triangles) as a function of the tip-sample separation. The tip was placed above the middle of the $20 \times 80 \mu \mathrm{m}^{2}$ sample. The solid lines in (a) are a spline fit to data to guide the eye.
TABLE I. The intensity ratios for modes $(1,3),(1,5),(1,7)$, and $(1,9)$ in the presence of a strong positive tip field $\left(h_{\text {tip }}>0\right)$, a weak tip field $\left(h_{\text {tip }} \approx 0\right)$, and a negative tip field $\left(h_{\text {tip }}<0\right)$. Corresponding spectra are plotted in Fig. 1.

\begin{tabular}{lccc}
\hline \hline & $I(1,3) / I(1,5)$ & $I(1,5) / I(1,7)$ & $I(1,7) / I(1,9)$ \\
\hline$h_{\text {tip }}>0$ & $0.6 \pm 0.1$ & $2.9 \pm 0.1$ & $1.0 \pm 0.2$ \\
$h_{\text {tip }} \approx 0$ & $1.6 \pm 0.1$ & $1.9 \pm 0.1$ & $1.5 \pm 0.2$ \\
$h_{\text {tip }}<0$ & $2.4 \pm 0.2$ & $1.1 \pm 0.3$ & $1.9 \pm 0.4$ \\
\hline \hline
\end{tabular}

line, and for strong tip fields, $h_{\text {tip }}>0$ and $h_{\text {tip }}<0$. The values in the first row are lower/higher/lower than values for $h_{\text {tip }}$ $\approx 0$ while the values in the third row are higher/lower/higher than the values for $h_{\text {tip }} \approx 0$. This reinforces the conclusions made earlier that for $h_{\text {tip }}>0$, the modes $(1,5)$ and $(1,9)$ are enhanced, while for $h_{\text {tip }}<0$ the modes $(1,3)$ and $(1,7)$ are enhanced.

The influence of the perturbation field of the probe magnet is further demonstrated in lateral scans taken along the long axis of the sample at $z \approx 3 \mu \mathrm{m}$. Figure 3(a) shows the experimental data of the force amplitudes for the $\left(1, n_{y}\right)$ modes as a function of position. Three interesting features are observed. (i) As the tip is moved from the center of the sample, the field of the tip magnet has broken the even symmetry of the internal field. As a result, the "hidden" $(1,2)$ mode is excited. (ii) For the $(1,3)$ mode, the intensity at $y$ $=0 \mu \mathrm{m}$ is strongly suppressed while at the position of the next maxima in the magnetostatic mode at $y= \pm 26 \mu \mathrm{m}$ the intensity is enhanced. (iii) Mode $(1,5)$ shows very little variation as a function of position of the tip. Therefore, the lateral resolution can be estimated to be better than $20 \mu \mathrm{m}$. This is in good agreement with the theoretical predictions [Fig. 3(b)].

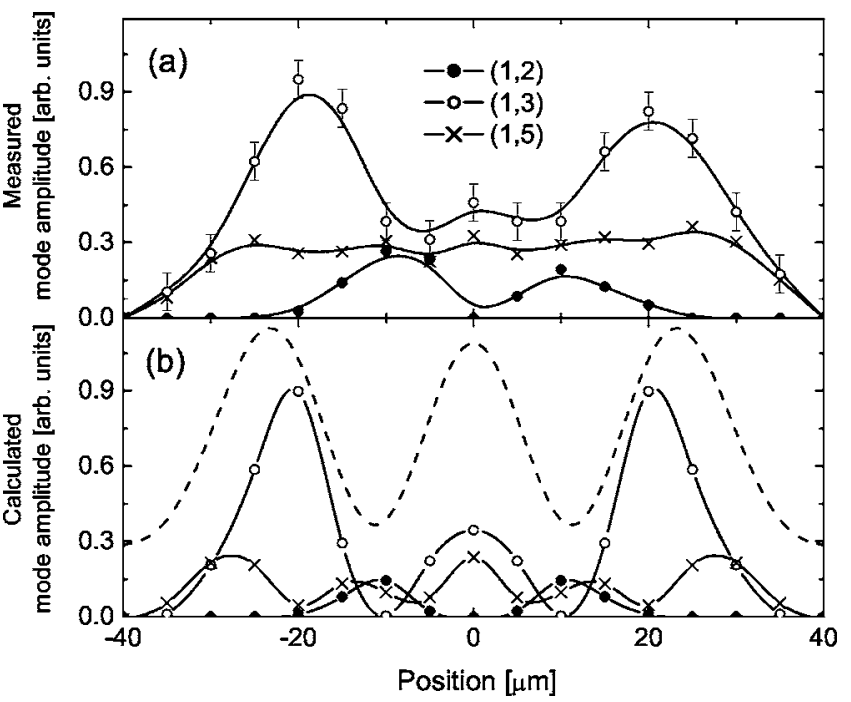

FIG. 3. The mode intensity for the $(1,2),(1,3)$, and $(1,5)$ modes as the tip is scanned along the long axis of the $20 \times 80 \mu \mathrm{m}^{2}$ sample. The external dc field and the probe field are parallel. The tip-sample separation $z \approx 3 \mu \mathrm{m}$. (a) Measured response. (b) Calculated amplitude. The dashed line represents the unperturbed $(1,3)$ mode with 0.3 offset. 
These unusual characteristics are understood within a theoretical model, which is based on a linearized LandauLifshitz-Gilbert (LLG) equation of motion that includes a spatially dependent tip field and Maxwell's equations. The external dc field is parallel to the sample normal, and the driving rf field, $h_{\mathrm{rf}}$, lies in the plane of the sample and is considered to be homogeneous over the entire sample. The cantilever tip field, $h_{\text {tip }}(\boldsymbol{r})$, is considered to be either parallel, $h_{\text {tip }}>0$, or antiparallel, $h_{\text {tip }}<0$, to the external field $\boldsymbol{H}_{\text {ext }}$.

The linearized LLG equation of motion and Maxwell's equations neglecting displacement currents can be written as (in cgs units)

$$
\begin{gathered}
\frac{\partial \boldsymbol{M}}{\partial t}=-\gamma\left[\boldsymbol{M} \times \boldsymbol{H}_{\mathrm{eff}}\right]+\frac{\alpha}{M_{s}}\left[\boldsymbol{M} \times \frac{\partial \boldsymbol{M}}{\partial t}\right], \\
\boldsymbol{\nabla} \times \boldsymbol{H}=\mathbf{0}, \quad \boldsymbol{\nabla} \cdot(\boldsymbol{H}+4 \pi \boldsymbol{M})=\mathbf{0},
\end{gathered}
$$

where $\gamma$ is the absolute value of gyromagnetic ratio, $M_{s}$ is the saturation magnetization, and $\alpha$ is the dimensionless Gilbert damping parameter. For our YIG samples, these values are $1.84 \times 10^{7} \mathrm{Oe}^{-1} \mathrm{~s}^{-1}, 140 \mathrm{erg} / \mathrm{cm}^{3}$, and 0.0045 , respectively. The internal effective field, $\boldsymbol{H}_{\mathrm{eff}}$, is given by

$$
\boldsymbol{H}_{\mathrm{eff}}=\left[h_{x}+h_{\mathrm{rf}}, h_{y}, H_{\mathrm{ext}}+H_{\mathrm{demag}}(\boldsymbol{r})+h_{\mathrm{tip}}(\boldsymbol{r})\right],
$$

where $H_{\text {demag }}(\boldsymbol{r})$ is a spatially dependent internal demagnetizing field. Equation (1) is solved in a small-angle approximation; $|\boldsymbol{m}| \ll M_{s}$, where $\boldsymbol{m}$ represents the transverse rf component of the magnetization vector. The boundary conditions are assumed to be

$$
\left.\boldsymbol{m}\right|_{x= \pm L_{x} / 2}=\left.\boldsymbol{m}\right|_{y= \pm L_{y} / 2}=0 .
$$

The unique feature in the model is treating the tip field as a nonlocal perturbation and determining its effect on the magnetostatic modes. Using Eq. (2), the rf magnetic field $\boldsymbol{h}$ is expressed in terms of the magnetization $\boldsymbol{m}(\boldsymbol{r})$ as a linear functional $\boldsymbol{h}[\boldsymbol{m}(\boldsymbol{r})]$ resulting in the nonlocal relationship

$$
\begin{gathered}
\hat{K} \boldsymbol{m}(\boldsymbol{r})+h_{0}(\boldsymbol{r}) \boldsymbol{m}(\boldsymbol{r})=\Delta \Omega \boldsymbol{m}(\boldsymbol{r})+h_{\mathrm{rf}}, \\
\Delta \Omega=\frac{1}{4 \pi M_{s}}\left(\frac{\omega}{\gamma}-H_{\mathrm{ext}}+4 \pi M_{s}\right), \\
h_{0}(\boldsymbol{r})=\frac{h_{\mathrm{tip}}(\boldsymbol{r})}{4 \pi M_{s}} .
\end{gathered}
$$

The operator $\hat{K}$ represents a linear integral transformation defined by

$$
\hat{K} f_{k}(\boldsymbol{r})=\omega_{k} f_{k}(\boldsymbol{r}),
$$

where $f_{k}$ represents the unperturbed magnetostatic modes given by

$$
f_{k}(x, y)=\sin \left\{k_{x}\left(x+L_{x} / 2\right)\right\} \sin \left\{k_{y}\left(y+L_{y} / 2\right)\right\}
$$

and $\omega_{k}$ is their dispersion relation given by the DamonEshbach theory. ${ }^{15}$ The wave vectors $\boldsymbol{k}$ are chosen to satisfy the "pinned" boundary conditions, Eq. (4). The new basis of

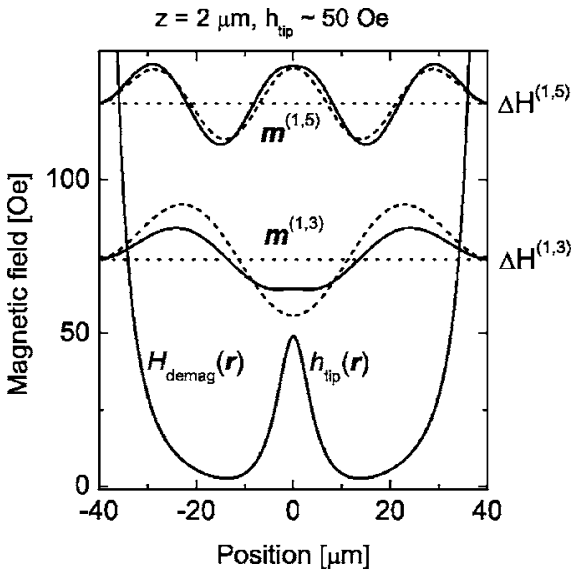

FIG. 4. The calculated magnetostatic modes of the 20 $\times 80 \mu \mathrm{m}^{2}$ sample with the tip located at the center of the film. The transverse component of magnetization $\boldsymbol{m}$ for unperturbed (dashed) and perturbed (solid) modes is plotted for $h_{\mathrm{tip}}=+50 \mathrm{G}$. The $\Delta H^{(1,3)}$ and $\Delta H^{(1,5)}$ represent the resonance field shift with respect to $\omega / \gamma$ $+4 \pi M_{s}$ for modes $(1,3)$ and $(1,5)$, respectively. Notice that the wavelength of the mode is increased in the vicinity of the tip. For the $(1,5)$ mode, this region increases the net dipole moment and therefore increases the FMRFM signal. For the $(1,3)$ mode, this region decreases the net dipole moment and therefore reduces the FMRFM signal.

the magnetostatic modes $m_{n}(\boldsymbol{r})$ can be found by solving the homogeneous equations

$$
\left[\hat{K}+h_{0}(\boldsymbol{r})\right] m_{n}(\boldsymbol{r})=\Delta \Omega_{n} m_{n}(\boldsymbol{r}) .
$$

The FMRFM signal is proportional to the force acting on the cantilever, which can be written as

$$
F_{\mathrm{FMRFM}} \approx d \int_{A} M_{z}(\boldsymbol{r}) \cdot \nabla_{z} h_{\mathrm{tip}}(\boldsymbol{r}) d x d y,
$$

where $M_{z}(\boldsymbol{r})=M_{s}-|\boldsymbol{m}|^{2} / 2 M_{s}$ is the longitudinal component of magnetization, $d$ is the thickness of the film, and $A$ represents the area of the sample.

Equation (10) offers an interesting interpretation; it is similar to the time-independent Schrödinger equation with the kinetic energylike term given by $\omega_{k}$ in Eq. (8). The normalized tip field $h_{0}(\boldsymbol{r})$ in Eq. (7) plays the role of a potential energy. When the tip field is parallel to the external field, $h_{0}(\boldsymbol{r})>0$, it creates a potential barrier for the magnetostatic modes, while $h_{0}(\boldsymbol{r})<0$ yields a potential well. This analogy provides qualitative insight into how the magnetostatic modes are modified by the tip magnetic field.

$h_{\text {tip }}>0$. The effect of the perturbation field when the tip field is parallel to the bias field is to increase the wavelength of the magnetostatic mode in the region near the tip. Figure 4 shows this effect when the tip is located at the center of the film. If $\boldsymbol{m}$ is positive (i.e., when $\boldsymbol{m}$ is parallel to the net dipole moment of the mode) in the region near the tip as in the case of mode $(1,5)$ shown in Fig. 4, the net dipole moment of the mode will be increased and therefore the FMRFM signal is enhanced. On the other hand, if $\boldsymbol{m}$ is negative (i.e., when $\boldsymbol{m}$ is antiparallel to the net dipole moment of the mode) in the 
region of the tip field as shown for mode $(1,3)$ in Fig. 4, the net dipole moment will be decreased and therefore the FMRFM signal for that mode is suppressed.

As the tip approaches the surface of the sample, the tip field gradient increases and therefore the FMRFM signal increases, see Fig. 2. However, in the region where the field separation of the magnetostatic mode $\Delta H^{\left(n_{x}, n_{y}\right)}$ is smaller than the maximum value of the tip field, $h_{\text {tip }}$, the mode becomes evanescent in that region. This decreases the amplitude of the magnetostatic wave in the vicinity of the tip magnet resulting in a reduced FMRFM signal even though the gradient of the field is increased. This is demonstrated by the decrease in the mode intensity as the tip approaches the sample, as shown in Fig. 2(b).

When the tip magnet is scanned across the sample, the transverse component of the magnetization $\boldsymbol{m}$ changes sign at the next lobe position; at $y=0 \mu \mathrm{m}$, the transverse component of $\boldsymbol{m}$ for the $(1,3)$ mode is negative and therefore the intensity of this mode is strongly suppressed. On the other hand, at $y= \pm 26 \mu \mathrm{m}, \boldsymbol{m}$ is positive and, as observed in Fig. 4 , this mode is strongly enhanced.

$h_{\text {tip }}<0$. In the case of the potential well, the results are exactly the opposite to those for a potential barrier $\left(h_{\text {tip }}\right.$ $>0$ ). If $\boldsymbol{m}$ is negative in the region of the tip magnet, as is the case for mode $(1,3)$, the wavelength of the wave in the region of the perturbing tip field is decreased and the coupling of the mode to the rf field is increased. Consequently, the FMRFM intensity of the mode is enhanced. On the other hand, if $\boldsymbol{m}$ is positive in the region of the tip field as for mode $(1,5)$, the net dipole moment will be decreased and therefore the FMRFM signal for that mode is suppressed.

Extending the Schrödinger equation analogy to the case of a deep narrow potential well, a highly localized magnetic mode can be excited. The properties of this localized mode would be independent of the sample size and therefore of any edge imperfections that might be introduced in the sample preparation. Scanning the local mode about the sample would allow one to extract variations in the local magnetic properties and damping parameter of the ferromagnetic samples. This makes FMRFM a unique experimental method allowing the investigation of local magnetic properties of the ferromagnetic samples independent of the sample dimensions, shape, and/or defects at the sample edge. Incorporation of advanced nanomagnetic probe tips should enable next-generation FMRFM systems capable of imaging local magnetic properties with submicrometer lateral resolution.

In conclusion, it has been demonstrated that the magnetic field of the tip magnet introduces a local inhomogeneity in the internal field of a $20 \times 80 \mu \mathrm{m}^{2}$ YIG film. This influences the shape of the samples magnetostatic modes, thereby measurably perturbing the strength of the force coupling to the cantilever. Using a hard magnetic coating on the tip, we are able to investigate the magnetostatic modes when the tip field is parallel $\left(h_{\text {tip }}>0\right)$ and antiparallel $\left(h_{\text {tip }}<0\right)$ to the external dc field. The condition $h_{\text {tip }}>0$ can be visualized as a potential barrier in the Schrödinger-like equation analogy, and modes $(1,5)$ and $(1,9)$ are enhanced while modes $(1,3)$ and $(1,7)$ were suppressed. In contrast with this is the case for $h_{\text {tip }}<0$ corresponding to a potential well in the Schrödinger-like equation analogy. Here the observed effect is exactly opposite to the case for $h_{\text {tip }}>0$. These results are in excellent agreement with the proposed theory, indicating that an inhomogeneity in the internal field can also be successfully included into FMRFM experiments.

We thank Melissa M. Midzor for assistance and discussions. We gratefully acknowledge financial support from ONR MURI under Grant No. DAAD 19-01-0541. P.E.W. was supported by the R.J. Yeh fund during the course of this research.
${ }^{1}$ B. Heinrich, Can. J. Phys. 78, 161 (2000).

${ }^{2}$ D. Rugar, C. Yannoni, and J. Sidles, Nature (London) 360, 563 (1992).

${ }^{3}$ P. C. Hammel, Z. Zhang, G. Moore, and M. L. Roukes, J. Low Temp. Phys. 271, 59 (1995).

${ }^{4}$ D. Rugar, R. Budakian, H. Mamin, and B. Chui, Nature (London) 430, 329 (2004).

${ }^{5}$ D. Rugar, O. Zuger, S. Hoen, C. S. Yannoni, H.-M. Vieth, and R. Kendrick, Science 264, 1560 (1994).

${ }^{6}$ Z. Zhang, P. C. Hammel, and P. E. Wigen, Appl. Phys. Lett. 68, 2005 (1996).

${ }^{7}$ M. Loehndorf, J. Moreland, and P. Kabos, Appl. Phys. Lett. 76, 1176 (2000).

${ }^{8}$ V. Charbois, V. Naletov, J. Youssef, and O. Klein, J. Appl. Phys.
91, 7337 (2002).

${ }^{9}$ A. Volodin, D. Buntinx, S. Brems, and C. van Haesendonck, Appl. Phys. Lett. 85, 5935 (2004).

${ }^{10}$ M. M. Midzor, P. E. Wigen, D. Pelekhov, W. Chen, P. C. Hammel, and M. L. Roukes, J. Appl. Phys. 87, 6493 (2000).

${ }^{11}$ M. M. Midzor, Ph.D. dissertation, California Institute of Technology (2001).

${ }^{12}$ O. Klein, V. Charbois, V. V. Naletov, and C. Fermon, Phys. Rev. B 67, 220407(R) (2003).

${ }^{13}$ S. Signoretti, C. Beeli, and S. Liou, J. Magn. Magn. Mater. 27276, 2167 (2004).

${ }^{14}$ P. E. Wigen, M. L. Roukes, and P. C. Hammel, Ferromagnetic Resonance Force Microscopy (Springer-Verlag, in press).

${ }^{15}$ J. Eshbach and R. Damon, Phys. Rev. 118, 1208 (1960). 